\title{
Terminating black holes in asymptotically free quantum gravity
}

\author{
Cosimo Bambi ${ }^{a}$, Daniele Malafarina ${ }^{b}$, Leonardo Modesto $^{c}$ \\ Department of Physics, Center for Field Theory and Particle Physics, Fudan University, Shanghai 200433, China
}

Received: 9 January 2014 / Accepted: 31 January 2014 / Published online: 20 February 2014

(C) The Author(s) 2014. This article is published with open access at Springerlink.com

\begin{abstract}
We study the homogeneous gravitational collapse of a spherical cloud of matter in a super-renormalizable and asymptotically free theory of gravity. We find a picture that differs substantially from the classical scenario. The central singularity appearing in classical general relativity is replaced by a bounce, after which the cloud re-expands indefinitely. We argue that a black hole, strictly speaking, never forms. The collapse only generates a temporary trapped surface, which can be interpreted as a black hole when the observational timescale is much shorter than the one of the collapse. However, it may also be possible that the gravitational collapse produces a black hole and that after the bounce the original cloud of matter evolves into a new universe.
\end{abstract}

\section{Introduction}

When a star exhausts all its nuclear fuel, the thermal pressure of its particles cannot compensate for the gravitational force any more, and the body contracts until it finds a new equilibrium configuration. For very massive stars, there is no known mechanism capable of compensating their own gravitational force, and the body will undergo a complete gravitational collapse. In general relativity, under the assumptions of the validity of the strong energy condition and of the existence of global hyperbolicity, the final product of the collapse is a singularity of the spacetime $[1,2]$. At the singularity, predictability is lost and standard physics breaks down. According to the weak cosmic censorship conjecture, singularities produced in the gravitational collapse must be hidden behind an event horizon and the final product of the collapse is a black hole $[3,4]$. The energy conditions and the cosmic censorship conjecture are two fundamental ingredients in the

\footnotetext{
a e-mail: bambi@fudan.edu.cn

be-mail: daniele@fudan.edu.cn

c e-mail: 1modesto@fudan.edu.cn
}

theory of black hole physics, as they regulate most of the properties of their horizons (see e.g. Ref. [2], chapter 9).

In this paper, we study the gravitational collapse in a large class of non-local theories of gravity, which also includes models inspired by string field theory [5-10]. We consider the simplest cases of homogeneous collapse of a spherical cloud of dust and radiation and we find a new picture for the gravitational collapse. The spacetime singularity appearing in classical general relativity is replaced by a bounce, after which the cloud re-expands indefinitely. It seems that black holes, strictly speaking, never form, in the sense that there are no regions causally disconnected from future null infinity. We find that the collapse produces a temporary trapped surface, which appears like the classical apparent horizon in the weak field regime and is removed when size and density lead to the regime of asymptotic freedom. The object can look like a black hole to far-away observers when the observational timescale is much shorter than the one of the collapse. In terms of the effective theory, in which the Einstein equations are satisfied by an effective perfect fluid matching at the boundary to a Vaidya solution, the disappearance of the horizon can be seen as the result of an ingoing flux of negative energy. One practical consequence of the model is that astrophysical black hole candidates should be characterized by an apparent mass loss, which might produce some observational effects. While we study in some detail only a specific model, we argue that this picture for the gravitational collapse must be common to many quantum-gravity theories. Independently of the exact UV completion, in several models quantum corrections make gravity repulsive at very high densities $[11,12]$, and this is the key-ingredient to get our result.

\section{Theoretical framework}

As classical action, we consider a 'non-polynomial' or 'semipolynomial' extension of Stelle's quadratic theory $[13,14]$, 
in which the dimensionless coupling constants are replaced by an entire function of the D'Alembertian operator [15-22]

$\mathcal{S}=\int \mathrm{d}^{4} x \frac{2 \sqrt{|g|}}{\kappa^{2}}\left[R-G_{\mu \nu} \frac{V\left(-\square / \Lambda^{2}\right)^{-1}-1}{\square} R^{\mu \nu}\right]$,

where $G_{\mu \nu}$ is the Einstein tensor and $\kappa^{2}=32 \pi G_{N}$. All the non-polynomiality is in the form factor $V\left(-\square / \Lambda^{2}\right)$, where $\Lambda$ is the Lorentz invariant energy scale. $\Lambda$ is not subject to infinite or finite (non analytic) renormalizations, and it is only constrained to be large by observations. The natural value of $\Lambda$ is of the order of the Planck mass. At the classical level, all the corrections to the Einstein-Hilbert action are suppressed by $1 / \Lambda$, and therefore the theory reduces to general relativity at low energies. That is also true at the quantum level, as a consequence of the Donoghue argument [23] (see Appendix A for more details).

The entire function $V\left(-\square / \Lambda^{2}\right)$ must have no poles in the whole complex plane, in order to ensure unitarity, and must exhibit at least logarithmic behavior in the ultraviolet regime, to give super-renormalizability at the quantum level. General form factors suggested by a class of theories consistent at the quantum level are

$$
\begin{aligned}
& V(z)^{-1}=\left|p_{\gamma+1}(z)\right| e^{\frac{1}{2}\left[\Gamma\left(0, p_{\gamma+1}^{2}(z)\right)+\gamma_{E}\right],} \\
& V(z)^{-1}=e^{z^{n}} \quad n \in \mathbb{N}^{+},
\end{aligned}
$$

where $z \equiv-\square / \Lambda^{2}$ and $p_{\gamma+1}(z)$ is a real polynomial of degree $\gamma+1(\gamma \in \mathbb{N}, \gamma>2)$. The theory is uniquely specified once the form factor is fixed, because the latter does not receive any renormalization: the ultraviolet theory is dominated by the bare action (that is, counterterms are negligible). In this class of theories, we only have the graviton pole. Since $V(z)$ is an entire function, there are no ghosts and no tachyons, independently of the number of time derivatives present in the action.

Concerning the difficulties with particular form factors and non-local operators, we note that the class of operators introduced by Krasnikov- $V(z)^{-1}$ as given by Eq. (3) with $n$ even - and the one introduced by Tomboulis $-V(z)^{-1}$ as given by Eq. (2) - are well defined in the Euclidean as well as in the Lorentzian case, because $\left(k_{\mathrm{E}}^{2}\right)^{2}=\left(k^{2}\right)^{2}$, where $k_{\mathrm{E}}$ is the momentum in the Euclidean space (see Refs. $[15,16]$ ). In the following, we concentrate on the case in Eq. (3) with $n=1$, which is suggested by string theory [5-10,24-26], but the qualitative behavior holds for a large class of models. In this paper we do all the calculations in the Lorentzian case, where the integral of interest is converging. To stress the genericity of the result, independently of the Wick rotation, we present the solution with $n=2$ in Appendix $C$ and we have checked the solutions for other even values of $n$. There are no qualitative differences for different values of $n$ in the physical quantities studied in this work.

\section{Homogeneous collapse}

The most general spherically symmetric metric describing a collapsing cloud of matter in comoving coordinates is given by

$\mathrm{d} s^{2}=-e^{2 v} \mathrm{~d} t^{2}+\frac{R^{\prime 2}}{G} \mathrm{~d} r^{2}+R^{2} \mathrm{~d} \Omega^{2}$,

where $\mathrm{d} \Omega^{2}$ represents the line element on the unit two-sphere and $\nu, R$, and $G$ are functions of $t$ and $r$. In the homogeneous marginally bound case, we can choose $v=0$ and $G=1$ (see e.g. Ref. [27]). The standard Einstein equations for the collapse of a perfect fluid are

$\frac{\kappa^{2}}{4} \rho=\frac{F^{\prime}}{R^{2} R^{\prime}}, \quad \frac{\kappa^{2}}{4} p=-\frac{\dot{F}}{R^{2} \dot{R}}$,

where the ' denotes a derivative with respect to $r$, and the - denotes a derivative with respect to $t$. Here $\rho$ and $p$ are, respectively, the density and the pressure of the fluid, while $F$ is the Misner-Sharp mass, which is defined by $F=R \dot{R}^{2}$ and turns out to be twice the total gravitational mass contained within the shell labeled by $r$ at the time $t$. In the case of collapse, the usual prescription is that the area radius $R(r, t)$ is set equal to the comoving radius $r$ at the initial time $t_{\mathrm{i}}=$ $0, R(r, 0)=r$. We can then introduce a scale factor $a(t)$, $R(r, t)=r a(t)$, with $a(0)=1$.

Let us first study the radiation case where $p=\rho / 3$. The classical solution is

$a(t)^{2}=\left|\frac{t_{0}-t}{t_{0}}\right|$,

where $t=t_{0}$ is the time of occurrence of the singularity. For the theory defined in Eq. (1), it is more convenient to find the solution of the scale factor with the propagator approach $[29,30]$, rather than by solving the counterpart of the Einstein equations (5). The procedure and the details of the calculations are reported in Appendix B. The final result is

$a^{2}(t)=\frac{2 e^{-\frac{1}{4} \Lambda^{2}\left(t-t_{0}\right)^{2}}}{\Lambda \sqrt{\pi} t_{0}}+\frac{\left(t_{0}-t\right) \operatorname{erf}\left(\frac{\Lambda\left(t_{0}-t\right)}{2}\right)}{t_{0}}$,

where $\operatorname{erf}(z)=2 \int_{0}^{z} \exp \left(-t^{2}\right) \mathrm{d} t / \sqrt{\pi}$. The classical singularity is now replaced by a bounce at $t=t_{0}$, as can be seen in the left panel of Fig. 1. In the spirit of Ref. [27], we can write the effective Einstein equations, in which $\rho$ and $p$ in Eq. (5) are replaced, respectively, by an effective density $\rho_{\text {eff }}$ and an effective pressure $p_{\text {eff }}$. The effective pressure is

$p_{\text {eff }}=-\frac{4}{\kappa^{2}}\left[\left(\frac{\dot{a}}{a}\right)^{2}+2 \frac{\ddot{a}}{a}\right]$.

$p_{\text {eff }}$ is close to the classical value $p=\rho / 3$ far from the time $t=t_{0}$, while it becomes negative around $t=t_{0}$. 

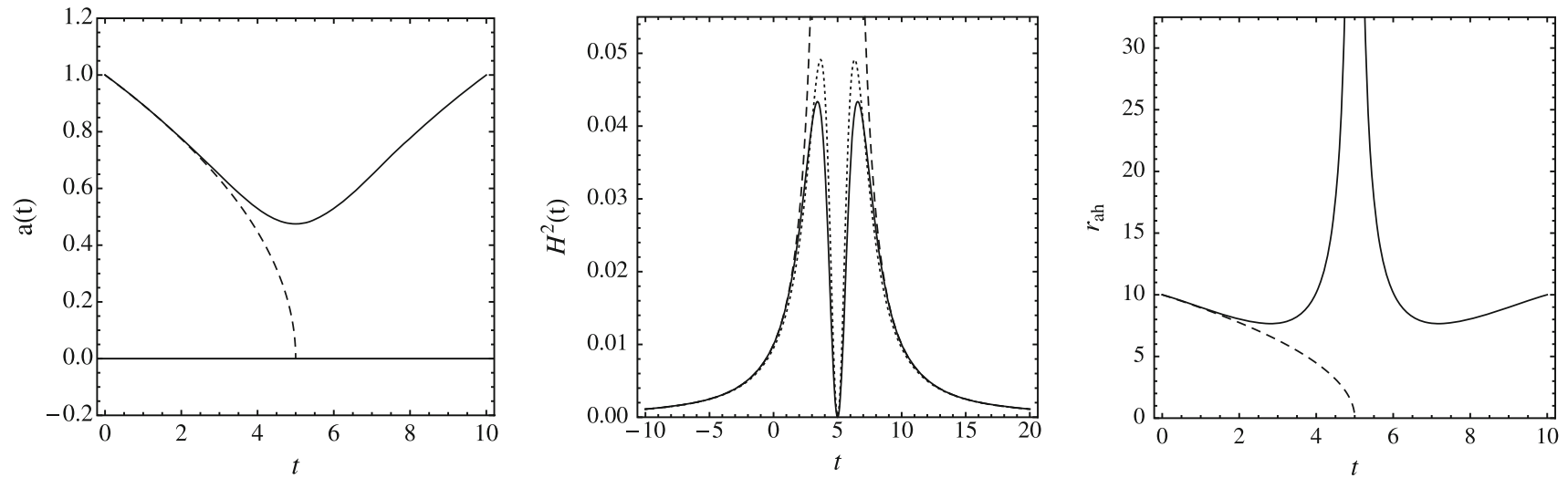

Fig. 1 Left panel evolution of the scale factor $a(t)$ for the classical radiation collapse (dashed curve) and the semiclassical radiation collapse (solid curve). Central panel as in the left panel for the square of the Hubble rate $H^{2}=(\dot{a} / a)^{2}$ and comparison with the effective radiation

Asymptotic freedom plays a crucial rule in the kind of approximation we are doing. It allows us to use only the two points function (propagator) because all the $n$-graviton interactions go to zero at high densities near the bounce. However, a generic asymptotic freedom is sufficient to remove the singularity, but it is not enough to have a bounce. Here, the asymptotic freedom is due to a higher derivative form factor, which makes gravity repulsive at very small distances. In particular, we would like to stress that the repulsion that causes the cloud to expand is not given by the quantum mechanical nature (Heisenberg uncertainty) of the collapsing matter in the regime in which gravity vanishes. The bounce follows from the dynamics of the system. In terms of the effective picture, the bounce comes from the conservation of the (effective) energy-momentum tensor: the matter is transformed into a state with $\rho_{\text {eff }}+p_{\text {eff }}<0$, which is unstable and therefore the bounce is the only available possibility.

The Hubble rate $H=\dot{a} / a$ is shown in the central panel of Fig. 1. It is interesting to compare this $H$ with the one we obtain from an effective theory in which we introduce an effective energy density expressed in terms of the radiation energy density $[27,31]$

$H^{2}:=\frac{\kappa^{2}}{12} \rho_{\mathrm{eff}}=\frac{\kappa^{2}}{12} \rho\left[1-\left(\frac{\rho}{\rho_{\mathrm{cr}}}\right)^{\alpha}\right]$,

where $\rho=\rho_{0} / a^{4}$ is the (physical) radiation energy density, $\rho_{0}=12 /\left(\kappa^{2} t_{0}^{2}\right.$ ), and $\alpha$ is a model-dependent parameter (for instance, $\alpha \approx 1$ in loop quantum cosmology [31]). When $\Lambda \sim M_{\mathrm{Pl}}$, we should expect that the critical density $\rho_{\mathrm{cr}}$ is of order the Planck energy density. The plot of $H^{2}(t)$ for $\alpha=1$ is shown in the central panel of Fig. 1. The modifications induced by quantum effects can essentially be incorporated in a new term proportional to $\rho^{2}$, which is negligible for $\rho \ll \rho_{\text {cr }}$ and becomes relevant as $\rho$ approaches $\rho_{\text {cr }}$. When model with $\alpha=1$ in Eq. (9) (dotted curve). Right panel evolution of the radius of the apparent horizon $r_{\mathrm{ah}}$ for the classical radiation collapse (dashed curve) and semiclassical radiation collapse (solid curve). Here $t_{0}=5$ and $\Lambda=1$. See the text for more details

$\rho=\rho_{\text {cr }}$, gravity is turned off, $H=0$, and we have the bounce.

The same procedure can be followed to study the collapse of dust. Now the classical solution is

$a(t)^{2}=\left|\frac{t_{0}-t}{t_{0}}\right|^{\frac{4}{3}}$.

The quantum-gravity-corrected solution is

$a^{2}(t)=-\frac{2 \Gamma\left(-\frac{2}{3}\right) \Gamma\left(\frac{4}{3}\right){ }_{1} F_{1}\left(-\frac{2}{3} ; \frac{1}{2} ;-\frac{\left(t_{0}-t\right)^{2} \Lambda^{2}}{4}\right)}{\Lambda^{4 / 3} \sqrt{3} \pi t_{0}^{4 / 3}}$,

where ${ }_{1} F_{1}$ is the Kummer confluent hypergeometric function. Just as in the radiation case, also in the case of dust matter there is a bounce at the time $t_{0}$ in place of a classical singularity.

\section{Trapped surfaces and Penrose diagram}

The condition for the formation of trapped surfaces is given by the requirement that the surface $R(r, t)=$ constant is null; that is, $g^{\mu \nu}\left(\partial_{\mu} R\right)\left(\partial_{\nu} R\right)=0$. In our homogeneous marginally bound collapse, this reduces to $1-\dot{R}^{2}=0$, and therefore

$r_{\mathrm{ah}}=\frac{1}{|\dot{a}|}$.

The right panel of Fig. 1 shows the evolution of the radius of the apparent horizon $r_{\mathrm{ah}}$ for the radiation model, both in the classical and quantum scenarios. In the classical case, there is no way to avoid the formation of the apparent horizon: the latter forms at the boundary of the collapsing cloud at a time $t<t_{0}$, before the formation of the singularity, and then propagates inwards to reach the center at the time of formation of the singularity. When the collapsing cloud crosses the 
Schwarzschild radius, the event horizon forms in the exterior spacetime and the formation of a black hole as the final stage of the collapse is indicated by the instant of formation of the trapped surfaces.

As shown in the right panel of Fig. 1, the semiclassical scenario is qualitatively different. In both the radiation and the dust collapses, the curve $r_{\mathrm{ah}}(t)$ is delayed with respect to the classical model and then reaches a minimum at a time $t_{*}$, where $\ddot{a}=0$ and $\dot{a}$ reaches a maximum value $\dot{a}\left(t_{*}\right)=\dot{a}_{*}$. This leads to the existence of a limiting radius $r_{*}$

$r_{*}=\frac{1}{\left|\dot{a}_{*}\right|}$.

If the boundary of the cloud is $r_{\mathrm{b}}<r_{*}$, then no trapped surface forms at any time during the collapse. We have thus a threshold mass, below which the collapsing matter can always be seen by a distant observer. Of course, this threshold is related to the scale introduced by the quantum theory, in our case by $\Lambda$, and therefore can be relevant for objects of planckian size.

Within this semiclassical scenario, it seems that a black hole, strictly speaking, never forms, in the sense that there is no region causally disconnected from future null infinity. The whole picture can be summarized as follows. At the beginning, the semiclassical collapse is close to the classical scenario. As the matter density increases, the gravitational force becomes weaker. In the language of the effective picture, quantum-gravity effects become important when the physical energy density approaches the critical one and the effective energy density goes to zero. In both the semiclassical radiation and the dust models, we have a bounce, after which the collapse turns into an expansion. Near the critical time of the bounce, gravity is weak (it is completely turned off at the time $t_{0}$ of the bounce) and any horizon disappears, at least in the interior solution, thus leaving the high density region potentially visible to distant observers. Such a possibility is eventually determined by the form and behavior of the exterior $r>r_{\mathrm{b}}$ spacetime, which we do not know for the full quantum-gravity theory under consideration. However, the semiclassical analogy, together with classical models matching to generalized Vaidya spacetimes with outgoing radiation, and some arguments related to the continuity of the trapping horizon suggest that the trapped region disappears also in the exterior. After the bounce, a new horizon forms, as a consequence of the decrease in the matter energy density and the increase of the gravity strength, and then disappears for ever at later times, when the radius of the apparent horizon exceeds $r_{\mathrm{b}}$. The formation/evaporation of the trapped surface is determined by the asymptotically free nature of gravity. The second trapped surface forms when gravity leaves the asymptotic freedom regime and it becomes strong again.

The Penrose diagram is shown in Fig. 2. Unlike the classical case where $t_{0}$ represents the singularity time, the con-

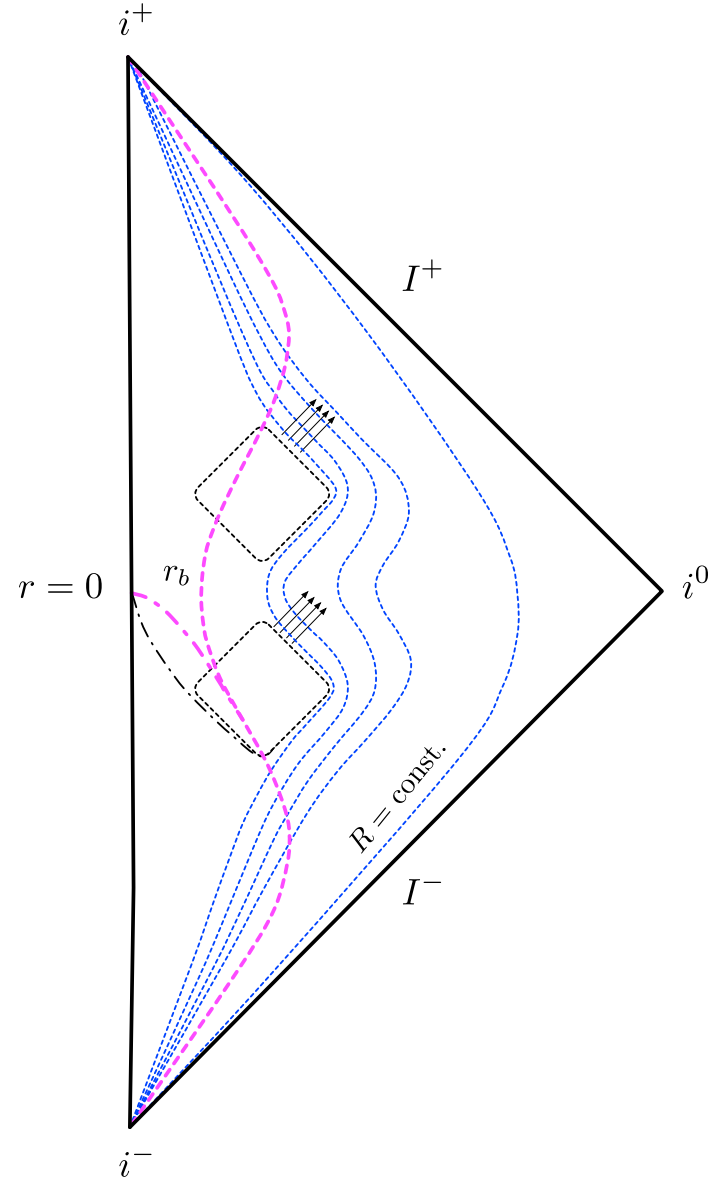

Fig. 2 Penrose diagram for the semiclassical radiation collapse model described by Eq. (7). The magenta thick-dashed line is the curve of the radius of the boundary of the collapsing object (the timeline curve defined by $R_{\mathrm{b}}(t)=r_{\mathrm{b}} a(t)$ ), while the black thin-dashed lines represent the trapped surface. In the classical model, there is no bounce; at a certain time, the behavior departs from the semiclassical solution, as shown by the dotted-dashed lines (magenta thick line for $r_{\mathrm{b}}$, black thin line for $\left.r_{\mathrm{ah}}\right)$. The blue-dotted lines are curves of constant radial coordinate

formal diagram for the collapse model extends to $t$ going to future infinity. In the semiclassical picture, the trapped region develops as in the classical regime, but it then disappears at the bounce due to the semiclassical corrections, and this is accompanied by a second trapped region in the corresponding expanding phase. Our theory has small departures from classical general relativity at low density/curvature and no superluminal motion. The key-point to understand the Penrose diagram, and in particular the destruction of the horizon, is the following. Interior homogeneous solutions matched with an exterior vacuum Schwarzschild spacetime hold in the special case of classical general relativity with a cloud of dust. Beyond general relativity, the Birkhoff theorem does not usually hold. However, we can recast the quantum-gravity theory in a semiclassical effective theory that describes a fluid that in general is not dust and violates the energy conditions. In the general case, therefore including in general relativity 
but without dust, the matching has to be done with a generalized Vaidya solution, which represents a spacetime with ingoing or outgoing null flux of energy. While here we have only the interior $r<r_{\mathrm{b}}$ metric, the external part in the effective picture is surely a Vaidya metric. In other words, our collapsing object presumably has a lot of 'hairs'. If we want to see the collapse in terms of the effective picture, in which the Einstein equations are satisfied by an effective fluid covering the whole spacetime, the exterior solution should be an ingoing flux of negative energy. It is this external ingoing flux that allows for the destruction of the horizon. As the theory has small deviations from classical general relativity at low energy density/curvature, it is clear that this flux is very low and therefore that the lifetime of the trapped surface-as measured by a distant observer-must be long for an astrophysical object with $M / \Lambda \gg 1$ (see next section for more details).

The above picture for gravitational collapse seems plausible, because the matching with the Vaidya exterior is a well known procedure and the interpretation appears quite natural. However, without knowing the exact form of the exterior solution, one cannot in principle exclude other scenarios. For example, the outward flux of energy may be irrelevant, thus leaving an exterior that is almost vacuum and therefore the gravitational collapse may produce an ordinary black hole with an almost constant mass $M_{\text {in }}$ given by the gravitational mass of the collapsing cloud. After the bounce, the expanding cloud would then be confined inside the Schwarzschild radius and would evolve into a new (expanding) universe. In this case, the Penrose diagram could look like one of those reported in Ref. [32].

Universes created as offspring of collapse to black holes have been considered in the literature [33-36]. This kind of scenario can generally be obtained analytically by means of a cut and paste procedure in which a singular manifold, such as the Schwarschild black hole, can be extended beyond the singularity by removing the same and sewing the spacetime to a new non singular manifold describing an expanding baby universe. However, as far as we are aware, even this procedure is feasible only in very simple examples and becomes highly non trivial if one wishes to consider the dynamical setup. Typically the matching involves continuity of the first and second fundamental forms across some hypersurface. To have the chance to fulfill these requirements one needs a large enough number of free parameters, which is not the case of marginally bound collapse.

Therefore, despite the general appeal that such a solution may have, one is faced with a lot of technical difficulties plus the important objection that the fact that a certain manifold can be constructed by hand does not imply necessarily that it is realistic. In this sense, an interior solution in the form of a scale factor $a$ obtained from a well posed theory and valid globally without any junction appears to be more natural choice. Indeed a selection principle as the one proposed by Smolin [37] is practically very difficult to achieve even if one neglects microphysics and considers only some effective theory of gravity as we do here.

\section{Astrophysical objects}

A model consistent with observations must be able to explain the super-massive black hole candidates in galactic nuclei, and therefore the trapped surface formed in the collapse for an object with mass $M \sim 10^{5}-10^{9} M_{\odot}$ must survive for a time at least comparable to the age of the Universe. For a heavy $(M / \Lambda \gg 1)$ astrophysical object, it is natural to expect that this is indeed the case, because the theory has small deviations from classical general relativity at low curvature and therefore the ingoing flux responsible for the destruction of the horizon must be very low; that is, the lifetime of the trapped surface must be long. For a comoving observer, the timescale of the collapse is of order the dynamical timescale $\kappa M \sim 1\left(M / 10^{6} M_{\odot}\right)$ s. For a distant observer, the timescale is longer, as a consequence of the gravitational redshift. The exact calculation of the lifetime of the trapped surface would require the knowledge of the metric in the whole spacetime, while in our case we have only the interior solution. An estimate of this time interval can be obtained from the velocity of shrinkage of the horizon $[11,12]$

$\sigma=\left(\frac{\mathrm{d} r}{\mathrm{~d} v}\right)_{g_{00}=0}$,

where $v=t+r$ is the advanced time and $g_{00}$ is the temporal component of the metric, while $g_{00}=0$ defines the apparent horizon. The shrinkage vanishes for the classical black hole case and the lifetime of the horizon is thus infinite in this case. As discussed in the previous section, one can also see the evaporation of the trapped surface in terms of an effective picture, in which the horizon is destroyed by an ingoing flux of negative energy. As we can play only with two mass scales, $\Lambda$ and $M$, we may guess that the order of magnitude is given by $\Lambda / M$, or that it is given by an expansion in $\Lambda / M$ and therefore even more suppressed. The lifetime of the trapped surface with respect to the distant observer is, in the more conservative case with $\mathrm{d} r / \mathrm{d} v \sim \Lambda / M$,

$\tau \sim \frac{\kappa M}{\mathrm{~d} r / \mathrm{d} v} \sim \kappa M\left(\frac{M}{\Lambda}\right)$,

which is anyway much longer than the age of the Universe (for the Sun, $M / \Lambda \sim 10^{38}$ if $\Lambda$ is of order the Planck mass).

Our theoretical model (homogeneous cloud of dust or radiation) is very simple, but it is easy to figure out how the picture might change in a more realistic scenario, at least 
qualitatively. It is natural to expect that the cloud is inhomogeneous, with a density profile monotonically decreasing in the outwards radial direction. Our results for the homogeneous case should hold along the central shell of the collapsing cloud, while at larger radii, since the density should be lower, one expects a smaller deviation from the standard general relativistic case. The bounce will remain, but at larger radii gravity may still be strong and therefore the instant of the bounce may not be visible to the distant observers (in the language of the Penrose diagram in Fig. 2, the two disconnected gray areas representing the trapped regions would be a single region).

An inevitable effect is an apparent mass loss of astrophysical black hole candidates. This is a consequence of asymptotic freedom and, strictly speaking, does not require the presence of the bounce. In other words, the only necessary ingredient is that gravity becomes very weak at high densities, while a negative effective pressure is not strictly necessary. As it is more clear in terms of the effective Einstein equations, the effective density decreases when the physical density approaches the critical one and increases after the bounce. The gravitational mass seen by a distant observer, i.e. $F_{\text {eff }}\left(r_{\mathrm{b}}, t\right) / 2$ where $r_{\mathrm{b}}$ is the boundary, does the same (at the bounce $\dot{a}=0$, so $\rho_{\text {eff }}=0$ ). A collapsing object should thus appear as a black hole candidate with a time varying mass. Such a prediction is robust, even if the exact behavior may depend on both the theoretical framework (e.g. the choice of the form factor) and the astrophysical content (matter equation of state, initial conditions, etc.), and it should be seen as an apparent mass loss of black hole candidates. If the apparent mass loss rate were to be relatively low and diluted for a long time, there might be a chance to observe it as an increase in the orbital period of a black hole binary. The future discovery of black hole binaries with a pulsar companion can presumably put the strongest constraints on such a possibility. On the other hand, if the apparent mass loss rate were high and for a relatively short time, resulting in a sudden disappearance of the object, the phenomenon may easily generate hypervelocity stars, i.e. stars which are observed with velocities of order $1,000 \mathrm{~km} / \mathrm{s}$ but whose origin is not yet clear [38]. Indeed, if the stellar companion were in a close high velocity orbit and could not feel the gravitational force of the black hole candidate for a while, it would escape with a velocity equivalent to its orbital velocity. The phenomenon may be particularly interesting to produce hypervelocity neutron stars.

\section{Summary and conclusions}

In this paper, we have studied the homogeneous collapse of a cloud in a super-renormalizable and asymptotically free theory of gravity. The final singularity of classical general relativity is removed and replaced by a bounce. Unitarity is necessary to have a 'good' theory, but it is irrelevant for the presence of the bounce, as can be seen in conformal gravity (which is asymptotically free, is not unitary, and predicts the bounce $[11,12])$. A generic asymptotic freedom is sufficient to remove the singularity, but it is not enough to have a bounce (see the case of QCD, where the asymptotic freedom is given by the matter content). The bounce requires a repulsive gravitational force at high densities. The key-point is therefore the form factor, which is related to the propagator and to the effective potential of the theory. Asymptotic freedom due to a higher derivative form factor introduces an effective negative pressure, which is responsible for the bounce. As in several quantum-gravity approaches corrections to classical general relativity make gravity repulsive at very high densities, independently of the exact UV completion, the prediction of the bounce is much more general and presumably holds in a larger class of theories. We indeed note that a bounce replacing the classical singularity in the gravitational collapse was previously found in different contexts $[39,40]$.

We argue that in these theories black holes, strictly speaking, never form. The Penrose diagram is shown in Fig. 2. The theory has small deviations from classical general relativity at low densities/curvature and no superluminal motion. The shrinkage of the external horizon is possible due to the matching of the effective solution describing the quantum corrections with a Vaidya spacetime in the exterior manifold. For massive astrophysical objects, deviations from general relativity are tiny and we can therefore expect that the lifetime of the trapped surfaces is long for an observer at infinity. Astrophysical black hole candidates may thus be objects with a temporary trapped surface, but they would be interpreted as black holes if the observational time scale is much shorter than the lifetime of the horizon. However, as we have derived only the interior solution in the full quantumgravity theory, we cannot really conclude that this is only possible scenario. The effect of the exterior Vaidya solution may very well be negligible in the dynamics of the collapse, even when integrated for a very long time. If this were to be the case, the collapse would produce a black hole with the usual Schwarzschild event horizon and the matter cloud, re-expanding after the bounce, would evolve into a new universe.

While our work neglects Hawking radiation, it, however, suggests a simple way to resolve the information paradox. In the first scenario, the information is trapped inside the apparent horizon and released when the latter eventually evaporates. It is an example of a 'complete evaporation scenario' according to the terminology of Ref. [41]. In the second case with a new universe, the spacetime decomposes into two regions and the information is stored in the new universe. This is the 'baby universe solution' [41]. 
Acknowledgments We thank Antonino Marciano for reading a preliminary version of this manuscript and providing useful feedback. This work was supported by the NSFC Grant No. 11305038, the Innovation Program of Shanghai Municipal Education Commission grant No. 14ZZ001, the Thousand Young Talents Program, and Fudan University.

Open Access This article is distributed under the terms of the Creative Commons Attribution License which permits any use, distribution, and reproduction in any medium, provided the original author(s) and the source are credited.

Funded by $\mathrm{SCOAP}^{3}$ / License Version CC BY 4.0.

\section{Appendix A: Ultraviolet and infrared properties of the theory}

We consider a particular representative theory of the following generic class:

$\mathcal{L}=2 \kappa^{-2} \sqrt{|g|}\left[R-G_{\mu \nu} \frac{V\left(-\square / \Lambda^{2}\right)^{-1}-1}{\square} R^{\mu \nu}\right]$,

where $G_{\mu \nu}$ is the Einstein tensor and $V\left(-\square / \Lambda^{2}\right)$ is an entire function. At the classical level, all the corrections to the Einstein-Hilbert action are suppressed by $1 / \Lambda$. Since $\Lambda$ is expected to be of order the Planck mass, at low energies the theory reduces to the Einstein one. At quantum level, the introduction of non-local operators in the action could leads to strong non-localities generated by the renormalization group flow toward the infrared, in disagreement with observations. This is not the case for the Lagrangian in Eq. (16), as we can see from the Donoghue argument [23].

The Donoghue result can be summarized as follows. If we start from a general covariant theory of gravity involving a Taylor expandable classical action, at quantum level we find analytical as well as non-analytical finite universal contributions coming from one-loop diagrams. If only massless particles are propagating, around the flat spacetime the non-analytical contribution has the form

$\kappa^{2} k^{2} \log \left(-k^{2}\right)$.

If we couple the theory to massive particles, we also have

$\kappa^{2} k^{2} \sqrt{\frac{m^{2}}{-k^{2}}}$

The analytical contributions are instead integer powers of the momentum $k$. This result also applies to our theory because the action involves only entire functions. The logarithmic non-analytic contribution to the one-loop effective action is related to the quantum corrections due to long distance effects of massless particles. In QED, one can see something similar in photon vacuum polarization,

$k^{2} \Pi\left(k^{2}\right) \sim k^{2}\left(\frac{1}{6 \epsilon}-\frac{\gamma_{E}}{6}+f(k)\right)$.
The other finite contributions to the one-loop amplitude are a series of analytic and sub-leading operators in the infrared regime $k \rightarrow 0$,

$\lim _{k \rightarrow 0} \sum_{n} R^{n}=0$

where $R$ is a general local curvature invariant. On the other hand, the non-analytic contribution (17) is divergent in the IR and gives corrections to the gravitational potential [23]. We find the same situation for photon vacuum polarization in QED if we take the limit of zero electron mass. The finite nonanalytic contributions coming from the divergent integrals in a massless theory and in our theory read

$$
\begin{aligned}
& \int \frac{\mathrm{d}^{4-\epsilon} k}{p^{2}(p-k)^{2}}=\Gamma(\epsilon / 2)\left(-k^{2}\right)^{\epsilon / 2} \\
& =\left(\frac{2}{\epsilon}-\gamma_{E}+O(\epsilon)\right)\left(1-\frac{\epsilon}{2} \ln \left(-k^{2} / \mu^{2}\right)+O\left(\epsilon^{2}\right)\right) \\
& =\frac{2}{\epsilon}-\ln \frac{-k^{2}}{\mu^{2}}+O(\epsilon) \\
& =\log \frac{\Lambda_{\mathrm{UV}}^{2}}{\mu^{2}}-\ln \frac{-k^{2}}{\mu^{2}}+O(\epsilon) \equiv-\ln \left(\frac{-k^{2}}{\Lambda_{\mathrm{UV}}^{2}}\right)+O(\epsilon) .
\end{aligned}
$$

Finite contributions are analytical operators $O\left(1 / l^{4}\right)$ in $D=$ 4 (for example $R^{2} \sim 1 / l^{4}$ ) and thus they do not affect the infrared theory even in our theory because only entire functions are present in the action. In general, the relevant quantities to get the one-loop effective action are [28]

$\operatorname{Tr} \ln \hat{\square},\left.\quad \nabla_{\mu_{1}} \ldots \nabla_{\mu_{p}} \frac{\hat{1}}{\square^{n}} \delta(x, y)\right|_{y=x}$.

In the coincidence limit, the logarithmic divergent contributions of the universal quantities (22) have the structure of (21). Other possible finite contributions instead are analytical and polynomial in the momentum.

In our higher derivative theory, the ultraviolet behavior is different with respect to the Einstein theory and it depends on the details of the effective action. The renormalization group has a non-linear behavior going from the particular ultraviolet regime associated with our regularized theory to the universal Einstein regime in the infrared. The Donoghue result shows that the infrared modifications are independent of the nature of the fundamental higher derivative theory and thus equivalent to those of the Einstein theory, Eqs. (17) and (18).

So far we have been generic. However, the theory is uniquely specified once the form factor is fixed, because it does not receive any renormalization at the quantum level. In other words, the ultraviolet theory is dominated by the bare action. For simplicity, let us assume the following Tomboulis form factor: 
$V^{-1}(z)=e^{H(z)}=\left|z^{\gamma+1}\right| e^{\frac{1}{2}\left[\Gamma\left(0, z^{2 \gamma+2}\right)+\gamma_{E}\right]}$.

The theory is therefore completely specified and the asymptotic behavior in the ultraviolet regime reads

$\mathcal{L}_{\mathrm{UV}} \approx \frac{2 \kappa^{-2} e^{\gamma_{\mathrm{E}} / 2}}{\Lambda^{2 \gamma+2}}\left(\frac{1}{2} R \square^{\gamma} R-R_{\mu \nu} \square^{\gamma} R^{\mu \nu}\right)$,

which depends only on the integer exponent $\gamma$. In this paper, we used the form factor suggested by string field theory to make easy the classical analysis. However, the main result of the paper is insensitive to the details of the theory.

The form factor $V(z)$ must have no extra poles in the whole complex plane, but it is also constrained to have a renormalizable or finite theory in the ultraviolet regime. This leaves us with a class of theories, each of them super-renormalizable because of the following reasons:

1. only a finite number of couplings is renormalized,

2. only a finite number of diagrams is divergent.

On the other hand, at the phenomenological level the form factor could be experimentally constrained, for example measuring the corrections to the gravitational potential, or hypothetically measuring a cross section in a scattering process at high energy. Since $V(z)$ is an entire function, there are no ghosts and no tachyons, independently of the number of time derivatives present in the action. This is the main reason to introduce a non-polynomial Lagrangian.

Concerning the Lorentz invariant scale $\Lambda$, there is no fine tuning and it is not subject to infinite or finite (non-analytic) renormalizations. It is only constrained to be a large mass scale by astrophysical or cosmological observations and it is natural to expect $\Lambda$ to be of the order of the Planck mass. As a consequence of (21) and (22), in the ultraviolet regime the one-loop corrections to the classical theory read

$R \ln (-\square) R$ and $R_{\mu \nu} \ln (-\square) R^{\mu \nu}$.

There are no finite renormalizations of $\Lambda$ because all the finite and infinite corrections to the operators $R \square^{n} R$ and $R_{\mu \nu} \square^{n} R^{\mu \nu}$ are absorbed in the running couplings of the theory and not in the scale $\Lambda$. However, these operators do not move the poles in the propagator, because they are suppressed by the form factor $V(z)$.

\section{Appendix B: Solution of the scale factor via the propagator approach}

The energy-momentum tensor in comoving coordinates for the generic spherically symmetric metric describing collapse given in Eq. (4) is given by

$T_{v}^{\mu}=\operatorname{diag}\left\{\rho(r, t), p_{r}(r, t), p_{\theta}(r, t), p_{\theta}(r, t)\right\}$.
Einstein's equations relate the metric functions to the matter content and are given by

$$
\begin{aligned}
\frac{\kappa^{2}}{4} p_{r} & =-\frac{\dot{F}}{R^{2} \dot{R}}, \quad \frac{\kappa^{2}}{4} \rho=\frac{F^{\prime}}{R^{2} R^{\prime}}, \\
v^{\prime} & =2 \frac{p_{\theta}-p_{r}}{\rho+p_{r}} \frac{R^{\prime}}{R}-\frac{p_{r}^{\prime}}{\rho+p_{r}}, \\
\dot{G} & =2 \frac{v^{\prime}}{R^{\prime}} \dot{R} G,
\end{aligned}
$$

where the ' denotes a derivative with respect to $r$, and the denotes a derivative with respect to $t$. The function $F(r, t)$ is called the Misner-Sharp mass, and in general it is

$F=R\left(1-G+e^{-2 v} \dot{R}^{2}\right)$.

In the homogeneous marginally bound case, from the first of Eq. (27) it follows that $F$ is a function of $r$ only and the matching to the exterior vacuum Schwarzschild spacetime is always possible. Furthermore Eq. (28) reduces to $v^{\prime}=0$ and we can always choose the time coordinate in such a way that $v=0$. Integration of Eq. (29) is then trivial and gives $G=1+f(r)$ and in the marginally bound collapse case we shall take the free integration function $f$ to be zero. The system is then fully specified once a gauge is fixed for the scale. This is usually done by fixing the scale at the initial time. It is common to define $R(r, t)=r a(t)$ with $a(0)=1$, so that to solve the system we only need to find the scale factor $a(t)$ by solving the corresponding field equations. Here, we use instead the propagator approach of Refs. [29,30].

We first write the metric as a flat Minkowski background plus a fluctuation $h_{\mu \nu}$,

$$
\begin{aligned}
& g_{\mu \nu}=\eta_{\mu \nu}+\kappa h_{\mu \nu}, \\
& \mathrm{d} s^{2}=\mathrm{d} t^{2}-a(t)^{2} \mathrm{~d} x^{i} \mathrm{~d} x^{j} \delta_{i j},
\end{aligned}
$$

where $\eta_{\mu \nu}=\operatorname{diag}(1,-1,-1,-1)$. The conformal scale factor $a(t)$ and the fluctuation $h_{\mu \nu}(t, \vec{x})$ are related by the following relations $[29,30]$ :

$$
\begin{aligned}
& a^{2}(t)=1-\kappa h(t), h\left(t=t_{0}\right)=0, g_{\mu \nu}\left(t=t_{0}\right)=\eta_{\mu \nu} \\
& h_{\mu \nu}(t, \vec{x})=h(t) \operatorname{diag}\left(0, \delta_{i j}\right) \equiv h(t) \mathcal{I}_{\mu \nu} .
\end{aligned}
$$

After a gauge transformation, we can rewrite the fluctuation in the usual harmonic gauge

$h_{\mu \nu}(x) \rightarrow h_{\mu \nu}^{\prime}(x)=h_{\mu \nu}(x)+\partial_{\mu} \xi_{v}+\partial_{\nu} \xi_{\mu}$,

$\xi_{\mu}(t)=-\frac{3 \kappa}{2} \operatorname{diag}\left(\int_{0}^{t} h\left(t^{\prime}\right) \mathrm{d} t^{\prime}, 0,0,0\right)$.

The fluctuation now reads

$h_{\mu \nu}^{\prime}(t, \vec{x})=h(t) \operatorname{diag}\left(-3, \delta_{i j}\right)$,

$h_{\mu}^{\prime \mu}(t, \vec{x})=-6 h(t)$. 
We can then switch to the standard gravitational 'barred' field $\bar{h}_{\mu \nu}^{\prime}$ defined by

$\bar{h}_{\mu \nu}^{\prime}=h_{\mu \nu}^{\prime}-\frac{1}{2} \eta_{\mu \nu} h_{\lambda}^{\prime \lambda}=-2 h(t) \mathcal{I}_{\mu \nu}$,

satisfying $\partial^{\mu} \bar{h}_{\mu \nu}^{\prime}=0$. The Fourier transform of $\bar{h}_{\mu \nu}^{\prime}$ is

$\tilde{\bar{h}}_{\mu \nu}^{\prime}(E, \vec{p})=-2 \tilde{h}(E)(2 \pi)^{3} \delta^{3}(\vec{p}) \mathcal{I}_{\mu \nu}$.

The classical solution for the cosmological scenario in the radiation fluid model is

$a(t)^{2}=\left|t / t_{0}\right|$,

where $t=0$ is the singularity time. With the solution (37), we can compute the Fourier transform $\tilde{h}(E)$ defined in (36)

$\tilde{h}(E)=\frac{2}{\kappa t_{0} E^{2}}+\frac{2 \pi}{\kappa} \delta(E)$.

We can obtain the same solution (38) from the classical propagator if we properly introduce a dimensionless fictitious source in the momentum space. We can then extend this procedure to the theory defined in Eq. (16) $[29,30]$. The gauge independent part of the graviton propagator for the theory (16) and energy tensor $\tilde{T}^{\rho \sigma}(p)$ is (see e.g. Ref. [17])

$$
\begin{gathered}
\mathcal{O}_{\mu \nu \rho \sigma}^{-1}(p)=\frac{V\left(p^{2} / \Lambda^{2}\right)}{p^{2}}\left(P_{\mu \nu \rho \sigma}^{(2)}-\frac{1}{2} P_{\mu \nu \rho \sigma}^{(0)}\right) \\
\Longrightarrow \bar{h}_{\mu \nu}^{\prime}(x)=\kappa \int \frac{\mathrm{d}^{4} p}{(2 \pi)^{4}} \mathcal{O}_{\mu \nu \rho \sigma}^{-1}(p) \tilde{T}^{\rho \sigma}(p) e^{i p x}
\end{gathered}
$$

where $P_{\mu \nu \rho \sigma}^{(2)}$ and $P_{\mu \nu \rho \sigma}^{(0)}$ are the graviton projectors,

$$
\begin{aligned}
& P_{\mu \nu \rho \sigma}^{(2)}=\frac{1}{2}\left(\theta_{\mu \rho} \theta_{\nu \sigma}+\theta_{\mu \sigma} \theta_{\nu \rho}\right)-\frac{1}{3} \theta_{\mu \nu} \theta_{\rho \sigma}, \\
& P_{\mu \nu \rho \sigma}^{(0)}=\frac{1}{3} \theta_{\mu \nu} \theta_{\rho \sigma},
\end{aligned}
$$

and $\theta_{\mu \nu}=\eta_{\mu \nu}-k_{\mu} k_{\nu} / k^{2}$. Therefore

$$
\begin{aligned}
h(t) & =\kappa \int \frac{\mathrm{d}^{4} p}{(2 \pi)^{4}} \frac{1}{p^{2} V^{-1}\left(p^{2} / \Lambda^{2}\right)} \tilde{\rho}(E, \vec{p}) e^{i p x} \\
& =\kappa \int \frac{\mathrm{d} E}{2 \pi} \frac{1}{E^{2} V^{-1}\left(E^{2} / \Lambda^{2}\right)} \tilde{\rho}(E) e^{i E t} .
\end{aligned}
$$

For $V\left(p^{2} / \Lambda^{2}\right)=1$, we recover the classical case and the solution for $h(t)$ is exactly (37) if we use the distribution

$$
\tilde{\rho}(E, \vec{p})=\underbrace{\left(\frac{2}{\kappa^{2} t_{0}}+\frac{2 \pi}{\kappa^{2}} E^{2} \delta(E)\right)}_{\tilde{\rho}(E)}(2 \pi)^{3} \delta^{3}(\vec{p}) .
$$

We can then use this distribution for the form factor $V(z)^{-1}=$ $e^{z}$. The fluctuation resulting from the integral (41) now becomes

$\kappa h(t)=1-a^{2}(t)=1-\frac{2 e^{-\frac{1}{4} \Lambda^{2} t^{2}}}{\Lambda \sqrt{\pi} t_{0}}-\frac{t \operatorname{erf}\left(\frac{\Lambda t}{2}\right)}{t_{0}}$, where $\operatorname{erf}(z)=2 \int_{0}^{z} \exp \left(-t^{2}\right) \mathrm{d} t / \sqrt{\pi}$. The solution is not a gauge artifact because we use the gauge independent projected propagator. Since we are interested in gravitational collapse rather than in the cosmological solution, we replace the time coordinate $t$ with $-t+t_{0}$ to have the classical singularity at $t=t_{0}$ and the initial time at $t=0$. So

$a^{2}(t)=\frac{2 e^{-\frac{1}{4} \Lambda^{2}\left(t-t_{0}\right)^{2}}}{\Lambda \sqrt{\pi} t_{0}}+\frac{\left(-t+t_{0}\right) \operatorname{erf}\left(\frac{\Lambda\left(-t+t_{0}\right)}{2}\right)}{t_{0}}$.

The same procedure can be followed to study the collapse of pressureless matter, i.e. dust. In the dust case, the classical solution for the cosmological scenario is given by

$a(t)^{2}=\left|t / t_{0}\right|^{\frac{4}{3}}$,

and the correct fictitious distribution to get the perturbative solution starting from the propagator reads

$\tilde{\rho}(E, \vec{p})=\left(\frac{4 \Gamma\left(\frac{4}{3}\right)}{\sqrt{3} t_{0}^{4 / 3}|E|^{\frac{1}{3}}}+2 \pi E^{2} \delta(E)\right) \frac{(2 \pi)^{3}}{\kappa^{2}} \delta^{3}(\vec{p})$,

where $\Gamma$ is the Euler gamma function. Using the distribution in (46), we can find the modified solution for the gravitational fluctuation (41). From $a^{2}(t)=1-\kappa h(t)$, we get

$$
a^{2}(t)=-\frac{2 \Gamma\left(-\frac{2}{3}\right) \Gamma\left(\frac{4}{3}\right){ }_{1} F_{1}\left(-\frac{2}{3} ; \frac{1}{2} ;-\frac{1}{4} t^{2} \Lambda^{2}\right)}{\Lambda^{4 / 3} \sqrt{3} \pi t_{0}^{4 / 3}},
$$

where ${ }_{1} F_{1}$ is the Kummer confluent hypergeometric function. The solution for the gravitational collapse scenario is obtained by replacing $t$ with $-t+t_{0}$, as is done for the radiation model.

\section{Appendix C: Models with form factor $\exp (-\square)^{n}$}

Finally, we have calculated some solutions for the entire function $V(z)^{-1}=e^{z^{n}}$ and higher even values of $n$. It turns out that all these models describe exactly the same physics, in the sense that they have the same qualitative behavior. The solutions are much more complicated. The case $n=2$ has homogeneous solutions

$$
\begin{aligned}
& a_{\text {radiation }}^{2}(t)=\left[2 \Gamma\left(\frac{3}{4}\right){ }_{1} F_{3}\left(-\frac{1}{4} ; \frac{1}{4}, \frac{1}{2}, \frac{3}{4} ; \frac{t^{4} \Lambda^{4}}{256}\right)\right. \\
& \left.+\Lambda^{2} t^{2} \Gamma\left(\frac{5}{4}\right){ }_{1} F_{3}\left(\frac{1}{4} ; \frac{3}{4}, \frac{5}{4}, \frac{3}{2} ; \frac{t^{4} \Lambda^{4}}{256}\right)\right] \frac{1}{\pi \Lambda t_{0}}, \\
& a_{\text {dust }}^{2}(t)=\frac{1}{3 \pi\left(\Lambda t_{0}\right)^{\frac{4}{3}}}\left[2 \pi{ }_{1} F_{3}\left(-\frac{1}{3} ; \frac{1}{4}, \frac{1}{2}, \frac{3}{4} ; \frac{t^{4} \Lambda^{4}}{256}\right)\right. \\
& \left.+\sqrt{3} \Lambda^{2} t^{2} \Gamma\left(\frac{1}{3}\right) \Gamma\left(\frac{7}{6}\right){ }_{1} F_{3}\left(\frac{1}{6} ; \frac{3}{4}, \frac{5}{4}, \frac{3}{2} ; \frac{t^{4} \Lambda^{4}}{256}\right)\right] .
\end{aligned}
$$



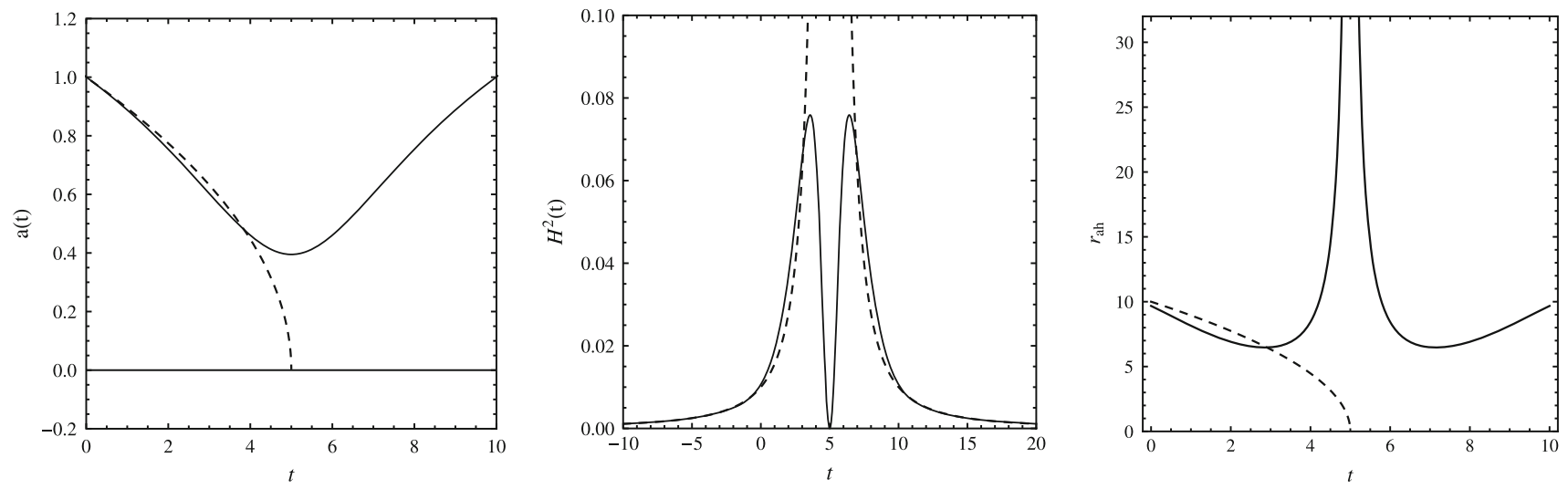

Fig. 3 Left panel evolution of the scale factor $a(t)$ for the classical radiation collapse (dashed curve) and the semiclassical radiation collapse (solid curve) in the case $n=2$. Central panel as in the left panel for the square of the Hubble rate $H^{2}=(\dot{a} / a)^{2}$. Right panel evolution of

The plots of the scale factor, Hubble rate, and apparent horizon are shown in Fig. 3. We have also checked the cases with $n=4$ and 12 , finding very similar plots.

\section{References}

1. S.W. Hawking, R. Penrose, Proc. R. Soc. Lond. A 314, 529 (1970)

2. S.W. Hawking, G.F.R. Ellis, The Large Scale Structure of SpaceTime (Cambridge University Press, Cambridge, 1973)

3. R. Penrose, Riv. Nuovo Cim. Numero Speciale 1, 252 (1969)

4. R. Penrose, Gen. Rel. Grav. 34, 1141 (2002)

5. V.A. Kostelecky, S. Samuel, Phys. Rev. D 42, 1289 (1990)

6. E. Witten, Nucl. Phys. B 268, 253-294 (1986)

7. D. Gross, E. Witten, Nucl. Phys. B 277, 1 (1986)

8. L. Brekke, P.G.O. Freund, M. Olson, E. Witten, Nucl. Phys. B 302, 365-402 (1988)

9. D.J. Gross, P.F. Mende, Phys. Lett. B 129 (1987)

10. A.S. Koshelev, [arXiv:1112.6410 [hep-th]]

11. V.P. Frolov, G.A. Vilkovisky, Phys. Lett. B 106, 307 (1981)

12. V.P. Frolov, G.A. Vilkovisky, IC-79-69 (1979)

13. K.S. Stelle, Phys. Rev. D 16, 953 (1977)

14. M. Asorey, J.L. Lopez, I.L. Shapiro, Int. J. Mod. Phys. A 12, 5711 (1997). [arXiv:hep-th/9610006]

15. N.V. Krasnikov, Theor. Math. Phys. 73, 1184 (1987)

16. N.V. Krasnikov, Teor. Mat. Fiz. 73, 235 (1987)

17. E.T. Tomboulis, [arXiv:hep-th/9702146]

18. L. Modesto, Phys. Rev. D 86, 044005 (2012). [arXiv:1107.2403 [hep-th]]

19. T. Biswas, E. Gerwick, T. Koivisto, A. Mazumdar, Phys. Rev. Lett. 108, 031101 (2012). [arXiv:1110.5249 [gr-qc]]

20. F. Briscese, A. Marciano, L. Modesto, E.N. Saridakis, Phys. Rev. D 87, 083507 (2013). [arXiv:1212.3611 [hep-th]] the radius of the apparent horizon $r_{\mathrm{ah}}$ for the classical radiation collapse (dashed curve) and semiclassical radiation collapse (solid curve). Here $t_{0}=5$ and $\Lambda=1$

21. S. Alexander, A. Marciano, L. Modesto, Phys. Rev. D 85, 124030 (2012). [arXiv:1202.1824 [hep-th]]

22. G.V. Efimov, Nonlocal Interactions of Quantized Fields [in Russian] (Nauka, Moscow, 1977)

23. J.F. Donoghue, Phys. Rev. D 50, 3874 (1994). [arXiv:gr-qc/ 9405057]

24. W. Siegel, [arXiv:hep-th/0309093]

25. T. Biswas, A. Mazumdar, W. Siegel, JCAP 0603, 009 (2006). [arXiv:hep-th/0508194]

26. T. Biswas, T. Koivisto, A. Mazumdar, JCAP 1011, 008 (2010). [arXiv:1005.0590v2 [hep-th]]

27. C. Bambi, D. Malafarina, L. Modesto, Phys. Rev. D 88, 044009 (2013). [arXiv:1305.4790 [gr-qc]]

28. A.O. Barvinsky and Vilkovisky, Phys. Rep. 119(1), 1-74 (1985)

29. M.J. Duff, Phys. Rev. D 9, 1837 (1974)

30. B. Broda, Phys. Rev. Lett. 106, 101303 (2011). [arXiv:1011.6257 [gr-qc]]

31. M. Bojowald, Living Rev. Rel. 11, 4 (2008)

32. D.G. Boulware, Phys. Rev. D 8, 2363 (1973)

33. V. Frolov, M. Markov, V. Mukhanov, Phys. Lett. B 216, 272 (1989)

34. V. Mukhanov, R. Brandenberger, Phys. Rev. Lett. 68, 1969 (1992)

35. R. Brandenberger, V. Mukhanov, A. Sornborger, Phys. Rev. D 48, 1629 (1993)

36. D.A. Easson, R.H. Brandenberger, JHEP 0106, 024 (2001). [arXiv: hep-th/0103019]

37. L. Smolin, Class. Quant. Grav. 9, 173 (1992)

38. W.R. Brown, M.J. Geller, S.J. Kenyon, Astrophys. J. 690, 1639 (2009). [arXiv:0808.2469 [astro-ph]]

39. R. Casadio, Int. J. Mod. Phys. D 9, 511 (2000). [arXiv:gr-qc/ 9810073]

40. R. Casadio, C. Germani, Prog. Theor. Phys. 114, 23 (2005). [arXiv: hep-th/0407191]

41. S. Hossenfelder, L. Smolin, Phys. Rev. D 81, 064009 (2010). [arXiv:0901.3156 [gr-qc]] 